\title{
Chemical stability of astaxanthin nanodispersions in orange juice and skimmed milk as model food systems.
}

\begin{abstract}
Solubilising astaxanthin in nanodispersion systems is a promising approach to incorporate astaxanthin into water-based food formulations. In this research, the chemical stabilities of astaxanthin nanodispersions diluted in orange juice and skimmed milk as model food systems and in deionised water as a control were evaluated. The nanodispersions displayed significantly $(\mathrm{p}<0.05)$ better stability in food systems compared to the control. The effects of stabilisers and dilution factor were also studied. In skimmed milk and deionised water, the type of stabiliser had a significant effect $(p<0.05)$ on astaxanthin degradation during storage. In vitro cellular uptake of astaxanthin from diluted astaxanthin nanodispersions in selected food systems was also evaluated. The cellular uptake of astaxanthin nanodispersions in skimmed milk was significantly higher $(\mathrm{p}<0.05)$ than that of astaxanthin nanodispersions in orange juice and deionised water. High in vitro cellular uptake of astaxanthin from the prepared astaxanthin nanodispersions can be achieved via incorporation into protein-based foods such as milk.
\end{abstract}

Keyword: Astaxanthin nanodispersions; Chemical stability; Bioavailability; Orange juice; Skimmed milk. 